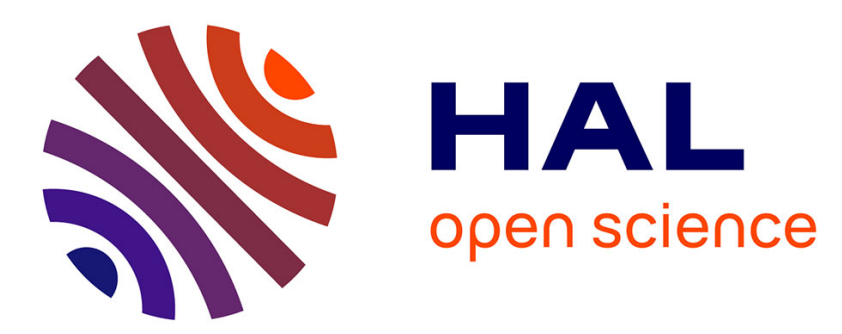

\title{
A note on convex ordering for stable stochastic integrals
}

\author{
Aldéric Joulin, Solym Manou-Abi
}

\section{To cite this version:}

Aldéric Joulin, Solym Manou-Abi. A note on convex ordering for stable stochastic integrals. Stochastics: An International Journal of Probability and Stochastic Processes, 2015, 87 (4), pp.592-603. 10.1080/17442508.2014.989528 . hal-02063817

\section{HAL Id: hal-02063817 https://hal.science/hal-02063817}

Submitted on 11 Mar 2019

HAL is a multi-disciplinary open access archive for the deposit and dissemination of scientific research documents, whether they are published or not. The documents may come from teaching and research institutions in France or abroad, or from public or private research centers.
L'archive ouverte pluridisciplinaire HAL, est destinée au dépôt et à la diffusion de documents scientifiques de niveau recherche, publiés ou non, émanant des établissements d'enseignement et de recherche français ou étrangers, des laboratoires publics ou privés. 


\title{
A NOTE ON CONVEX ORDERING FOR STABLE STOCHASTIC INTEGRALS
}

\author{
ALDÉRIC JOULIN AND SOLYM MAWAKI MANOU-ABI
}

\begin{abstract}
We establish a convex ordering between stochastic integrals driven by strictly $\alpha$-stable processes with index $\alpha \in(1,2)$. Our approach is based on the forward-backward stochastic calculus for martingales together with a suitable decomposition of stable stochastic integrals.
\end{abstract}

\section{INTRODUCTION}

It is by now well-known that theoretical development of stochastic orders provides elegant and practical tools for comparison of random phenomena, motivated by various applications in financial mathematics, risk management and stochastic networks. One of the most relevant stochastic orders is the convex order. Given $X$ and $Y$ two integrable random variables, we say that $X$ is less than $Y$ in the convex order, and we write $X \leq_{\mathrm{cx}} Y$, if we have the inequality

$$
\mathbb{E}[\phi(X)] \leq \mathbb{E}[\phi(Y)],
$$

for all convex functions $\phi$ such that the expectations exist. Such a stochastic order has some nice properties, among others the stability under mixtures and under convolution. In particular, if $X$ and $Y$ are two integrable random variables such that $X \leq_{\mathrm{cx}} Y$, then they have the same mean and moreover,

$$
\mathbb{E}\left[(X-K)^{+}\right] \leq \mathbb{E}\left[(Y-K)^{+}\right], \quad K \in \mathbb{R},
$$

where $F^{+}$denotes the positive part of a given random variable $F$. The latter inequality reveals to be convenient for financial applications (option pricing, hedging) and is actually equivalent to another stochastic order commonly denoted $\leq_{\text {icx }}$, the so-called increasing convex order, for which $\phi$ is additionally assumed to be non-decreasing. Another interesting consequence of the convex ordering is to facilitate the computation of distances between distributions such as the stop-loss distances (including the famous $L^{1}$-Wasserstein distance) and the Zolotarev distances, see for instance [4]. Historically, the notion of convex comparison was first introduced by Hoeffding in [9, 10] to obtain quantitative tail estimates. We refer to the monographs [15, 19] for an extensive analysis of many stochastic orders and their potential applications, with precise credit and references for this large body of

2010 Mathematics Subject Classification. 60G52, 60H05, 60E15, 60G44.

Key words and phrases. Stable process, stable stochastic integral, convex ordering, forwardbackward stochastic calculus. 
work. From a dynamical point of view, the convex ordering has been investigated in the context of Markov processes and semimartingales. See for instance the paper [8] dealing with diffusions, with a special emphasis on the Black-Scholes model, and also [2, 3] for option pricing involving general semimartingales and Lévy processes. In all these papers, the key point is the so-called propagation of convexity property. Other references such as [17, 18] use techniques based on the comparability of the infinitesimal generators of Markov processes together with the stochastic monotonicity property. Recently, another fruitful method was considered in [13], namely the so-called forward-backward stochastic calculus for martingales, which was followed by a series of papers based on this technology, cf. [1, 6, 17, 5, 14]. This approach reveals to be convenient in the non-Markovian framework, when dealing with Brownian stochastic integrals or stochastic integrals driven by a compensated Poisson random measure, and might be extended without any additional effort to the case of stochastic integrals driven by a Lévy process. However, the underlying assumptions required to obtain these convex comparison results enforce the driving Lévy process to have bounded jumps, excluding for instance the important class of stable processes.

Following these observations, the aim of this note is to establish a convex ordering for the stochastic integral $\int_{0}^{T} K_{t} d Z_{t}$ driven by a real strictly $\alpha$-stable process $\left(Z_{t}\right)_{t \in[0, T]}$, where $T>0$ is a finite time horizon. We restrict our attention to the case $\alpha \in(1,2)$ since the convex ordering only makes sense for random variables with finite mean. Although this stable process has sample paths of infinite variation and unbounded jumps, the forward-backward stochastic calculus combined with a suitable representation of stable stochastic integrals allow us to show the convex comparison

$$
\int_{0}^{T} K_{t} d Z_{t} \leq \mathrm{cx} \int_{0}^{T} k(t) d Z_{t}
$$

where $k$ is some deterministic function bounding, in a particular sense, the predictable process $\left(K_{t}\right)_{t \in[0, T]}$.

The paper is organized as follows. In Section 2, we recall some basic material on stable processes and stable stochastic integrals. Then we state our main contribution of the present work, Theorem 2.1, in which we derive the convex ordering mentioned above. Our result is a special instance of Theorem 2.2, which is more general but less tractable for practical issues since it compares in the convex order forward and backward stable stochastic integrals. Our last part, Section 3, is devoted to the proofs of these two results, on the basis of the forward-backward stochastic calculus introduced in [13] together with the representation of stable stochastic integrals developed by Kallenberg in [12]. 


\section{Preliminaries AND MAin RESUlts}

Consider on a probability space $(\Omega, \mathcal{A}, \mathbb{P})$ a strictly $\alpha$-stable process $Z:=\left(Z_{t}\right)_{t \in[0, T]}$ where $T$ is a finite time horizon and $\alpha \in(1,2)$. Here, strict-stability means the following self-similarity property

$$
\left(Z_{k t}\right)_{t \in[0, T]} \stackrel{d}{=}\left(k^{1 / \alpha} Z_{t}\right)_{t \in[0, T]}
$$

where $k>0$ and the equality $\stackrel{d}{=}$ is understood in the sense of finite dimensional distributions. The characteristic function is of the form

$$
\varphi_{Z_{t}}(u)=\exp \left(t \int_{-\infty}^{+\infty}\left(e^{i u y}-1-i u y\right) \nu(d y)\right), \quad t \in[0, T]
$$

where $\nu$ stands for the Lévy measure defined on $\mathbb{R} \backslash\{0\}$ by

$$
\nu(d x):=\frac{d x}{|x|^{\alpha+1}}\left(c_{+} 1_{\{x>0\}}+c_{-} 1_{\{x<0\}}\right) .
$$

The parameters $c_{+}, c_{-}$above are non-negative with furthermore $c_{+}+c_{-}>0$. In the case $c_{+}=c_{-}>0$, the process is said to be symmetric. The centered process $Z$ has sample paths of infinite variation and unbounded jumps, and it is a pure-jump martingale with respect to its natural filtration $\mathcal{F}_{t}^{Z}:=\sigma\left(Z_{s}: s \in[0, t]\right), t \in[0, T]$. Denoting $\mathcal{F}^{Z}$ this filtration, we assume that it satisfies the usual hypothesis, that is, completeness and right-continuity. The Lévy-Itô decomposition is given by

$$
Z_{t}=b_{R} t+\int_{0}^{t} \int_{|x| \leq R} x(\mu-\sigma)(d s, d x)+\int_{0}^{t} \int_{|x|>R} x \mu(d s, d x), \quad t \in[0, T],
$$

where $R$ is some arbitrary positive truncation level (classically chosen to be 1 ) and $\mu$ is a Poisson random measure on $\mathbb{R}_{+} \times \mathbb{R}$ with intensity measure $\sigma(d t, d x)=$ $d t \otimes \nu(d x)$. Here $b_{R}$ is the drift parameter given by

$$
b_{R}:=-\int_{|x|>R} x \nu(d x)=-\frac{\left(c_{+}-c_{-}\right) R^{1-\alpha}}{\alpha-1} .
$$

Denote respectively by $Z^{R-}$ and $Z^{R+}$ the two independent Lévy processes defined by the integrals above. The first one has a compactly supported Lévy measure and is a square-integrable martingale with infinitely many jumps bounded by $R$ on each compact time interval, whereas the second one is an integrable compound Poisson process with jumps larger than $R$. Let $K$ be a $\mathcal{F}^{Z}$-predictable process belonging to $L^{2}(\Omega \times[0, T])$, i.e.,

$$
\int_{0}^{T} \mathbb{E}\left[K_{t}^{2}\right] d t<+\infty .
$$

Then the stable integral $X_{T}:=\int_{0}^{T} K_{s} d Z_{s}$ is well-defined as a stochastic integral with respect to the Lévy-Itô decomposition of $Z$, that is $X_{T}=A_{T}^{R}+X_{T}^{R-}+X_{T}^{R+}$ with

$$
A_{T}^{R}:=b_{R} \int_{0}^{T} K_{t} d t, \quad X_{T}^{R-}:=\int_{0}^{T} K_{t} d Z_{t}^{R-} \quad \text { and } \quad X_{T}^{R+}:=\int_{0}^{T} K_{t} d Z_{t}^{R+},
$$


the second aforementioned process being a square-integrable martingale whereas the two other integrals are constructed in the Lebesgue-Stieltjes sense. In particular $X_{T}$ is integrable and therefore the process $X$ given by

$$
X_{t}:=\mathbb{E}\left[X_{T} \mid \mathcal{F}_{t}^{Z}\right]=\int_{0}^{t} K_{s} d Z_{s}, \quad t \in[0, T],
$$

is a $\mathcal{F}^{Z}$-martingale.

Let us turn to the question we are interested in, namely to establish a convex ordering between stable stochastic integrals. When dealing with a symmetric stable process $Z$ and deterministic integrands, we can proceed directly as follows. Let $k, \tilde{k} \in L^{2}([0, T])$ be two functions such that $|k(t)| \leq|\tilde{k}(t)|$ for any $t \in[0, T]$. Then the stable integrals

$$
X_{T}:=\int_{0}^{T} k(t) d Z_{t} \quad \text { and } \quad \tilde{X}_{T}:=\int_{0}^{T} \tilde{k}(t) d Z_{t},
$$

have the same distribution as the random variables $Z_{\sigma_{T}}^{\prime}$ and $Z_{\tilde{\sigma}_{T}}^{\prime}$ respectively, where $Z^{\prime}$ is a copy of the process $Z$ and the quantities $\sigma_{T}$ and $\tilde{\sigma}_{T}$ are defined by

$$
\sigma_{T}:=\int_{0}^{T}|k(t)|^{\alpha} d t \quad \text { and } \quad \tilde{\sigma}_{T}:=\int_{0}^{T}|\tilde{k}(t)|^{\alpha} d t .
$$

Denoting $\mathcal{F}^{Z^{\prime}}$ the natural filtration of $Z^{\prime}$, Jensen's inequality entails

$$
\begin{aligned}
\mathbb{E}\left[\phi\left(X_{T}\right)\right] & =\mathbb{E}\left[\phi\left(Z_{\sigma_{T}}^{\prime}\right)\right] \\
& =\mathbb{E}\left[\phi\left(\mathbb{E}\left[Z_{\tilde{\sigma}_{T}}^{\prime} \mid \mathcal{F}_{\sigma_{T}}^{Z^{\prime}}\right]\right)\right] \\
& \leq \mathbb{E}\left[\mathbb{E}\left[\phi\left(Z_{\tilde{\sigma}_{T}}^{\prime}\right) \mid \mathcal{F}_{\sigma_{T}}^{Z^{\prime}}\right]\right] \\
& =\mathbb{E}\left[\phi\left(Z_{\tilde{\sigma}_{T}}^{\prime}\right)\right] \\
& =\mathbb{E}\left[\phi\left(\tilde{X}_{T}\right)\right],
\end{aligned}
$$

where $\phi$ is a convex function such that the expectations exist. In other words we obtain the convex ordering $X_{T} \leq_{\mathrm{cx}} \tilde{X}_{T}$. For instance, such a result might be used to obtain a convex ordering between stable driven Ornstein-Uhlenbeck processes. Indeed consider the following random variables

$$
X_{T}:=e^{-\theta T} X_{0}+\int_{0}^{T} e^{-\theta(T-t)} d Z_{t} \quad \text { and } \quad Y_{T}:=e^{-\kappa T} X_{0}+\int_{0}^{T} e^{-\kappa(T-t)} d Z_{t},
$$

where $Z$ is a symmetric $\alpha$-stable process independent of the centered initial value $X_{0}$, and $\theta, \kappa \in \mathbb{R}$ are two parameters such that $\theta \geq \kappa$. If we denote

$$
k(t):=e^{-\theta(T-t)} \quad \text { and } \quad \tilde{k}(t):=e^{-\kappa(T-t)}, \quad t \in[0, T],
$$

then we have $k(t) \leq \tilde{k}(t)$ for any $t \in[0, T]$ so that the previous convex ordering yields

$$
\int_{0}^{T} e^{-\theta(T-t)} d Z_{t} \leq \mathrm{cx} \int_{0}^{T} e^{-\kappa(T-t)} d Z_{t}
$$


Applying now the following classical property on the increasing convex order $\leq_{\text {icx }}$, cf. [15]:

$$
\text { if } \quad 0 \leq a \leq b \text { and } \mathbb{E}\left[X_{0}\right]=0 \text { then } a X_{0} \leq_{\text {icx }} b X_{0},
$$

then with the choices of $a:=e^{-\theta T}$ and $b:=e^{-\kappa T}$, we obtain the increasing convex ordering $X_{T} \leq_{\text {icx }} Y_{T}$ (after a slight modification of the argument emphasized to derive the stability by convolution of the order $\left.\leq_{\text {icx }}\right)$. Finally, since $\mathbb{E}\left[X_{T}\right]=\mathbb{E}\left[Y_{T}\right]$, the order $X_{T} \leq_{\mathrm{icx}} Y_{T}$ thus implies the convex order $X_{T} \leq \mathrm{cx} Y_{T}$.

In the case of non-deterministic integrands, the story is more delicate, as it is already the case for Brownian stochastic integrals, cf. [8] and also the recent papers [5, 14] in which the authors overcome this difficulty by using the forward-backward stochastic calculus combined with the Malliavin calculus. In the stable setting we focus on in this note, we adapt the forward-backward stochastic calculus initially developed in [13] and use a convenient representation of stable stochastic integrals emphasized by Kallenberg in [12] to obtain the main result of this note. Since it is a particular case of the more abstract Theorem 2.2 which will be stated in a moment, we postpone the proof to Section 3. Recall that the notation $F^{+}$and $F^{-}$ stand respectively for the positive and negative parts of a given random variable or deterministic function $F$.

Theorem 2.1. Let $Z$ be a strictly stable process with index $\alpha \in(1,2)$. Let $K \in$ $L^{2}(\Omega \times[0, T])$ be a predictable process with respect to the natural filtration of $Z$ and let $k \in L^{2}([0, T])$. Denote for any $t \in[0, T]$ the rates

$$
\begin{array}{lll}
\gamma_{t}:=c_{+}^{1 / \alpha} K_{t}^{+}+c_{-}^{1 / \alpha} K_{t}^{-} & \text {and } & \tilde{\gamma}_{t}:=c_{+}^{1 / \alpha} k^{+}(t)+c_{-}^{1 / \alpha} k^{-}(t), \\
\lambda_{t}:=c_{+}^{1 / \alpha} K_{t}^{-}+c_{-}^{1 / \alpha} K_{t}^{+} & \text {and } & \tilde{\lambda}_{t}:=c_{+}^{1 / \alpha} k^{-}(t)+c_{-}^{1 / \alpha} k^{+}(t),
\end{array}
$$

and assume that they satisfy the a.s. conditions:

$$
\gamma_{t} \leq \tilde{\gamma}_{t} \quad \text { and } \quad \lambda_{t} \leq \tilde{\lambda}_{t}, \quad t \in[0, T] .
$$

Then the following convex ordering between stable stochastic integrals holds:

$$
\int_{0}^{T} K_{t} d Z_{t} \leq c x \int_{0}^{T} k(t) d Z_{t}
$$

Certainly, if the two inequalities in the assumptions are reversed, then the convex ordering is also reversed, as expected. Moreover, note that our result might be applied, among others, in the following two classical situations:

○ the process $Z$ is symmetric and a.s. $\left|K_{t}\right| \leq|k(t)|$ for any $t \in[0, T]$;

- both $K$ and $k$ are of the same constant sign and satisfy a.s. $K_{t} \leq k(t)$ for any $t \in[0, T]$.

For practical issues, we mention that the convex comparison result above is available for all convex functions $\phi$ whose growth at infinity is at most polynomial 
of degree $p$, with $1 \leq p<\alpha$. For instance, letting the convex function $\phi(x):=|x|^{p}$ where $1 \leq p<\alpha$, we need to verify that

$$
\mathbb{E}\left[\left|\int_{0}^{T} K_{t} d Z_{t}\right|^{p}\right]<+\infty
$$

so that we will obtain, under the assumptions of Theorem 2.1, the following inequality:

$$
\mathbb{E}\left[\left|\int_{0}^{T} K_{t} d Z_{t}\right|^{p}\right] \leq \mathbb{E}\left[\left|\int_{0}^{T} k(t) d Z_{t}\right|^{p}\right] .
$$

Let us proceed as in [11] by using a convenient truncation level $R$ in the Lévy-Itô decomposition. Using the notation introduced previously with $X_{T}:=\int_{0}^{T} K_{t} d Z_{t}$, we have for any fixed $x>0$,

$$
\mathbb{P}\left(\left|X_{T}\right|>x\right) \leq \mathbb{P}\left(\left|A_{T}^{R-}\right|>x / 2\right)+\mathbb{P}\left(\left|X_{T}^{R-}\right|>x / 2\right)+\mathbb{P}\left(X_{T}^{R+} \neq 0\right) .
$$

Denote $T_{1}^{R}$ the first jump time of the Poisson process $(\mu(\{y \in \mathbb{R}:|y|>R\} \times[0, t]))_{t \in[0, T]}$ on the set $\{y \in \mathbb{R}:|y|>R\}$. Since $T_{1}^{R}$ is exponentially distributed with parameter $\nu(\{y \in \mathbb{R}:|y|>R\})$, we have

$$
\begin{aligned}
\mathbb{P}\left(X_{T}^{R+} \neq 0\right) & \leq \mathbb{P}\left(T_{1}^{R} \leq T\right) \\
& \leq T \nu(\{y \in \mathbb{R}:|y|>R\}) \\
& =\frac{\left(c_{+}+c_{-}\right) T}{\alpha R^{\alpha}} .
\end{aligned}
$$

Now Chebyshev's inequality and the isometry formula for Poisson stochastic integrals entail

$$
\begin{aligned}
\mathbb{P}\left(\left|X_{T}^{R-}\right|>x / 2\right) & \leq \frac{4}{x^{2}} \mathbb{E}\left[\left|\int_{0}^{T} \int_{|y| \leq R} K_{t} y(\mu-\sigma)(d t, d y)\right|^{2}\right] \\
& =\frac{4}{x^{2}} \int_{|y| \leq R} y^{2} \nu(d y) \int_{0}^{T} \mathbb{E}\left[K_{t}^{2}\right] d t \\
& =\frac{4\left(c_{+}+c_{-}\right) R^{2-\alpha}}{(2-\alpha) x^{2}} \int_{0}^{T} \mathbb{E}\left[K_{t}^{2}\right] d t
\end{aligned}
$$

whereas by Markov's inequality,

$$
\begin{aligned}
\mathbb{P}\left(\left|A_{T}^{R}\right|>x / 2\right) & \leq \frac{2\left|b_{R}\right|}{x} \int_{0}^{T} \mathbb{E}\left[\left|K_{t}\right|\right] d t \\
& =\frac{2\left|c_{+}-c_{-}\right| R^{1-\alpha}}{(\alpha-1) x} \int_{0}^{T} \mathbb{E}\left[\left|K_{t}\right|\right] d t
\end{aligned}
$$


Therefore choosing the truncation level $R=x$ and rearranging the terms above entail the existence of some positive constant, say $M$, depending on all the parameters except $x$, such that $\mathbb{P}\left(\left|X_{T}\right|>x\right) \leq M x^{-\alpha}$. Finally, we obtain by integrating

$$
\begin{aligned}
\mathbb{E}\left[\left|X_{T}\right|^{p}\right] & =\int_{0}^{+\infty} p x^{p-1} \mathbb{P}\left(\left|X_{T}\right|>x\right) d x \\
& \leq 1+M \int_{1}^{+\infty} \frac{d x}{x^{\alpha-p+1}}
\end{aligned}
$$

which is finite since $p<\alpha$.

In order to state our second main contribution of the present note, let us recall the notion of backward processes. A backward strictly $\alpha$-stable process $\tilde{Z}$ is a strictly $\alpha$-stable process running backwards in time. It shares the same properties as the (forward) process $Z$ and in particular it is a backward martingale with respect to its own filtration. Indeed if $\mathcal{F}^{\tilde{Z}}$ denotes the complete and left-continuous filtration $\mathcal{F}_{t}^{\tilde{Z}}:=\sigma\left(\tilde{Z}_{s}: t \leq s \leq T\right)$, then the backward martingale property is satisfied, that is

$$
\mathbb{E}\left[\tilde{Z}_{s} \mid \mathcal{F}_{t}^{\tilde{Z}}\right]=\tilde{Z}_{t}, \quad 0 \leq s \leq t \leq T .
$$

For instance the process $\tilde{Z}$ defined by

$$
\tilde{Z}_{t}:=Z_{T-t}, \quad \text { with } \quad \mathcal{F}_{t}^{\tilde{Z}}:=\mathcal{F}_{T-t}^{Z}, \quad t \in[0, T],
$$

is a backward strictly $\alpha$-stable process. For the sake of briefness, the (forward) processes considered in the sequel are assumed to be right-continuous with left limits, whereas the backward processes are naturally supposed to be left-continuous with right limits. Now let $\tilde{K}$ be a $\mathcal{F}^{\tilde{Z}}$-predictable process belonging to $L^{2}(\Omega \times$ $[0, T])$. Then the backward stable integral $\tilde{X}_{0}:=\int_{0}^{T} \tilde{K}_{s} \tilde{d} \tilde{Z}_{s}$ is defined similarly to the forward case, the backward Itô differential $\tilde{d}$ meaning that the limit in probability is taken over sums of the type

$$
\sum_{i=1}^{p_{n}} \tilde{H}_{t_{i}^{n}}\left(\tilde{Z}_{t_{i-1}^{n}}-\tilde{Z}_{t_{i}^{n}}\right)
$$

for refining subdivisions $\left(t_{i}^{n}\right)$ of $[0, T]$. The random variable $\tilde{X}_{0}$ is integrable and thus the backward process $\tilde{X}$ given by

$$
\tilde{X}_{t}:=\mathbb{E}\left[\tilde{X}_{0} \mid \mathcal{F}_{t}^{\tilde{Z}}\right]=\int_{t}^{T} \tilde{K}_{s} \tilde{d} \tilde{Z}_{s}, \quad t \in[0, T],
$$

is a $\mathcal{F}^{\tilde{Z}}$-martingale.

Now we are in position to state our second result on which Theorem 2.1 is based. In order to relieve the presentation, the proof is also postponed to Section 3.

Theorem 2.2. Let $Z, \tilde{Z}$ be two independent forward and backward strictly stable processes, respectively, both with the same index $\alpha \in(1,2)$. Denote $c_{+}, c_{-}$and $\tilde{c}_{+}, \tilde{c}_{-}$the rates of the respective Lévy measures $\nu$ and $\tilde{\nu}$. Let $K, \tilde{K} \in L^{2}(\Omega \times[0, T])$ 
be two predictable processes with respect to the natural filtrations of $Z$ and $\tilde{Z}$, respectively. Denote for any $t \in[0, T]$ the rates

$$
\begin{array}{llll}
\gamma_{t}:=c_{+}^{1 / \alpha} K_{t}^{+}+c_{-}^{1 / \alpha} K_{t}^{-} & \text {and } & \tilde{\gamma}_{t}:=\tilde{c}_{+}^{1 / \alpha} \tilde{K}_{t}^{+}+\tilde{c}_{-}^{1 / \alpha} \tilde{K}_{t}^{-}, \\
\lambda_{t}:=c_{+}^{1 / \alpha} K_{t}^{-}+c_{-}^{1 / \alpha} K_{t}^{+} & \text {and } & \tilde{\lambda}_{t}:=\tilde{c}_{+}^{1 / \alpha} \tilde{K}_{t}^{-}+\tilde{c}_{-}^{1 / \alpha} \tilde{K}_{t}^{+},
\end{array}
$$

and assume that they satisfy the a.s. conditions:

$$
\gamma_{t} \leq \tilde{\gamma}_{t} \quad \text { and } \quad \lambda_{t} \leq \tilde{\lambda}_{t}, \quad t \in[0, T] .
$$

Then we have the following convex ordering between forward and backward stable stochastic integrals:

$$
\int_{0}^{T} K_{t} d Z_{t} \leq c x \int_{0}^{T} \tilde{K}_{t} \tilde{d} \tilde{Z}_{t}
$$

Our result remains in the spirit of the following well-known fact: if $Z^{\prime}$ is an independent copy of $Z$ and $\tilde{Z}$ is the backward process $\tilde{Z}_{t}=Z_{T-t}^{\prime}, t \in[0, T]$, then the distribution of the random variable $Z_{t}+\tilde{Z}_{t}$ does not depend on time $t \in[0, T]$ and thus has the same distribution as $Z_{T}$ and $\tilde{Z}_{0}$.

Before turning to the proofs, let us mention that Theorem 2.2 might be potentially extended. Indeed, up to some slight changes in the proof below, we are able to consider stable stochastic integrals for which a drift term is added, at the price of weakening the convex order $\leq_{\mathrm{cx}}$ by the increasing convex order $\leq_{\mathrm{icx}}$. Under the assumptions and notation of Theorem 2.2, consider the drifted integrals

$$
\int_{0}^{T} K_{t} d Z_{t}+\int_{0}^{T} L_{t} d t \quad \text { and } \quad \int_{0}^{T} \tilde{K}_{t} \tilde{d} \tilde{Z}_{t}+\int_{0}^{T} \tilde{L}_{t} d t
$$

where the processes $L, \tilde{L}$ are respectively $\mathcal{F}^{Z}$ - and $\mathcal{F}^{\tilde{Z}}$-adapted and satisfy the a.s. inequality $L_{t} \leq \tilde{L}_{t}$ for any $t \in[0, T]$. Then one needs in the forthcoming proof of Theorem 2.2 to assume that the convex functions of interest are also nondecreasing to preserve the order of the drift parts, so that we obtain the increasing convex ordering

$$
\int_{0}^{T} K_{t} d Z_{t}+\int_{0}^{T} L_{t} d t \leq \mathrm{icx} \int_{0}^{T} \tilde{K}_{t} \tilde{d} \tilde{Z}_{t}+\int_{0}^{T} \tilde{L}_{t} d t .
$$

In particular under appropriate assumptions, the strict stability of the processes $Z, \tilde{Z}$ might be relaxed since any integrable stable process can be transformed into a strictly stable process by removing its expectation (thus corresponding to a drift term).

\section{Proofs}

The core of the present part is devoted to the proof of Theorem 2.2 (the proof of Theorem 2.1 will follow immediately). Before entering into the details, let us concentrate first our attention on two important tools that will be used in the remainder of the paper, namely the forward-backward stochastic calculus developed 
in [13] and the decomposition of stable stochastic integrals due to Kallenberg [12]. In the sequel we work under the assumptions and notation of Theorem 2.2 .

Define the enlarged forward and backward filtrations $\mathcal{F}:=\left(\mathcal{F}_{t}\right)_{t \in[0, T]}$ and $\tilde{\mathcal{F}}:=$ $\left(\tilde{\mathcal{F}}_{t}\right)_{t \in[0, T]}$ as

$$
\mathcal{F}_{t}:=\mathcal{F}_{t}^{Z} \vee \mathcal{F}_{0}^{\tilde{Z}} \quad \text { and } \quad \tilde{\mathcal{F}}_{t}:=\mathcal{F}_{T}^{Z} \vee \mathcal{F}_{t}^{\tilde{Z}}, \quad t \in[0, T],
$$

where we recall that $\mathcal{F}^{Z}$ and $\mathcal{F}^{\tilde{Z}}$ are the natural filtrations generated by the processes $Z$ and $\tilde{Z}$, respectively. Then $Z$ is still a $\mathcal{F}$-martingale which is $\tilde{\mathcal{F}}$-adapted whereas $\tilde{Z}$ is a $\tilde{\mathcal{F}}$-martingale which is $\mathcal{F}$-adapted. In particular the processes $X$ and $\tilde{X}$ defined by

$$
X_{t}:=\int_{0}^{t} K_{s} d Z_{s} \quad \text { and } \quad \tilde{X}_{t}:=\int_{t}^{T} \tilde{K}_{s} \tilde{d} \tilde{Z}_{s}, \quad t \in[0, T],
$$

inherit the properties of $Z$ and $\tilde{Z}$, respectively. Let $\Delta X_{u}:=X_{u}-X_{u-}$ be the jump of $X$ at time $u \in(0, T]$ and define similarly the backward jump of $\tilde{X}$ at time $u \in[0, T)$ by $\tilde{\Delta} \tilde{X}_{u}:=\tilde{X}_{u}-\tilde{X}_{u+}$. Following [13], we recall Itô's formula for forwardbackward martingales, applied in our context to the pure-jump stable stochastic integrals $X, \tilde{X}$ : for any real-valued function $f \in \mathscr{C}^{1}\left(\mathbb{R}^{2}\right)$ and any $0 \leq s \leq t \leq T$,

$$
\begin{aligned}
f\left(X_{t}, \tilde{X}_{t}\right)= & f\left(X_{s}, \tilde{X}_{s}\right)+\int_{s^{+}}^{t} \frac{\partial f}{\partial x_{1}}\left(X_{u-}, \tilde{X}_{u}\right) d X_{u}-\int_{s}^{t^{-}} \frac{\partial f}{\partial x_{2}}\left(X_{u}, \tilde{X}_{u+}\right) \tilde{d} \tilde{X}_{u} \\
& +\sum_{s<u \leq t}\left(f\left(X_{u-}+\Delta X_{u}, \tilde{X}_{u}\right)-f\left(X_{u-}, \tilde{X}_{u}\right)-\Delta X_{u} \frac{\partial f}{\partial x_{1}}\left(X_{u-}, \tilde{X}_{u}\right)\right) \\
& -\sum_{s \leq u<t}\left(f\left(X_{u}, \tilde{X}_{u+}+\tilde{\Delta} \tilde{X}_{u}\right)-f\left(X_{u}, \tilde{X}_{u+}\right)-\tilde{\Delta} \tilde{X}_{u} \frac{\partial f}{\partial x_{2}}\left(X_{u}, \tilde{X}_{u+}\right)\right),
\end{aligned}
$$

where $\frac{\partial f}{\partial x_{i}}$ is the partial derivative of the function $f$ with respect to the $i^{\text {th }}$ coordinate, $i \in\{1,2\}$.

Now let us introduce the decomposition of stable stochastic integrals emphasized by Kallenberg in [12. Since the following argument can be easily generalized to the backward case, we will only focus our attention on the forward situation. First, note that the Lévy measure $\nu$ of the strictly $\alpha$-stable process $Z$ is of the form

$$
\nu=c_{+} \nu_{+}+c_{-} \nu_{-},
$$

where $\nu_{+}$and $\nu_{-}$are the measures on $\mathbb{R}_{+}$and $\mathbb{R}_{-}$, respectively, with Lebesgue density $|x|^{-\alpha-1}$. Hence the self-similarity entails trivially the following representation

$$
Z=c_{+}^{1 / \alpha} Z^{\prime}-c_{-}^{1 / \alpha} Z^{\prime \prime}
$$

where $Z^{\prime}, Z^{\prime \prime}$ are two independent strictly $\alpha$-stable processes with Lévy measure $\nu_{+}$. In other words, this decomposition is obtained by separating the positive and 
negative jumps of $Z$. Therefore the process $X$ can be rewritten as

$$
\begin{aligned}
X_{t} & =\int_{0}^{t} c_{+}^{1 / \alpha} K_{s} d Z_{s}^{\prime}-\int_{0}^{t} c_{-}^{1 / \alpha} K_{s} d Z_{s}^{\prime \prime} \\
& =\int_{0}^{t} c_{+}^{1 / \alpha} K_{s}^{+} d Z_{s}^{\prime}+\int_{0}^{t} c_{-}^{1 / \alpha} K_{s}^{-} d Z_{s}^{\prime \prime}-\int_{0}^{t} c_{+}^{1 / \alpha} K_{s}^{-} d Z_{s}^{\prime}-\int_{0}^{t} c_{-}^{1 / \alpha} K_{s}^{+} d Z_{s}^{\prime \prime} .
\end{aligned}
$$

Then Kallenberg's decomposition for the stable stochastic integral $X$ stands as follows:

$$
X_{t}=\int_{0}^{t} \gamma_{s} d \hat{Z}_{s}^{\prime}-\int_{0}^{t} \lambda_{s} d \hat{Z}_{s}^{\prime \prime}
$$

where the processes $\hat{Z}^{\prime}, \hat{Z}^{\prime \prime}$ given by the stable stochastic integrals

$$
\hat{Z}_{t}^{\prime}=\int_{0}^{t} 1_{\left\{K_{s} \geq 0\right\}} d Z_{s}^{\prime}+\int_{0}^{t} 1_{\left\{K_{s}<0\right\}} d Z_{s}^{\prime \prime} \quad \text { and } \quad \hat{Z}_{t}^{\prime \prime}=\int_{0}^{t} 1_{\left\{K_{s}<0\right\}} d Z_{s}^{\prime}+\int_{0}^{t} 1_{\left\{K_{s} \geq 0\right\}} d Z_{s}^{\prime \prime},
$$

are independent and have the same distribution as $Z^{\prime}, Z^{\prime \prime}$.

As announced above, we are now in position to prove Theorem 2.2 .

Proof of Theorem 2.2. Our aim is to establish the inequality

$$
\mathbb{E}\left[\phi\left(\int_{0}^{T} K_{t} d Z_{t}\right)\right] \leq \mathbb{E}\left[\phi\left(\int_{0}^{T} \tilde{K}_{t} \tilde{d} \tilde{Z}_{t}\right)\right],
$$

for any convex function $\phi$ such that the expectations exist. Since this inequality rewrites as

$$
\mathbb{E}\left[\phi\left(X_{T}+\tilde{X}_{T}\right)\right] \leq \mathbb{E}\left[\phi\left(X_{0}+\tilde{X}_{0}\right)\right],
$$

the proof will be achieved once we have established that the function $t \in[0, T] \mapsto$ $\mathbb{E}\left[\phi\left(X_{t}+\tilde{X}_{t}\right)\right]$ is non-increasing. At the price of an unessential regularizing procedure, we can assume without loss of generality that the convex function $\phi$ is also $\mathscr{C}^{1}$ and Lipschitz. Using then the Itô formula above with $f(x, y)=\phi(x+y)$, we have

$$
\begin{aligned}
\phi\left(X_{t}+\tilde{X}_{t}\right)= & \phi\left(X_{s}+\tilde{X}_{s}\right)+\int_{s^{+}}^{t} \phi^{\prime}\left(X_{u-}+\tilde{X}_{u}\right) d X_{u}-\int_{s}^{t^{-}} \phi^{\prime}\left(X_{u}+\tilde{X}_{u+}\right) \tilde{d} \tilde{X}_{u} \\
& +J_{s, t}^{X}-J_{s, t}^{\tilde{X}}
\end{aligned}
$$

where $J_{s, t}^{X}$ and $J_{s, t}^{\tilde{X}}$ are defined by

$$
\begin{aligned}
& J_{s, t}^{X}:=\sum_{s<u \leq t}\left(\phi\left(X_{u-}+\Delta X_{u}+\tilde{X}_{u}\right)-\phi\left(X_{u-}+\tilde{X}_{u}\right)-\Delta X_{u} \phi^{\prime}\left(X_{u-}+\tilde{X}_{u}\right)\right), \\
& J_{s, t}^{\tilde{X}}:=\sum_{s \leq u<t}\left(\phi\left(X_{u}+\tilde{X}_{u+}+\tilde{\Delta} \tilde{X}_{u}\right)-\phi\left(X_{u}+\tilde{X}_{u+}\right)-\tilde{\Delta} \tilde{X}_{u} \phi^{\prime}\left(X_{u}+\tilde{X}_{u+}\right)\right) .
\end{aligned}
$$


By Kallenberg's decomposition of the stable stochastic integral $X$, we can rewrite $J_{s, t}^{X}$ in the following way:

$$
\begin{aligned}
J_{s, t}^{X}= & \sum_{s<u \leq t}\left(\phi\left(X_{u-}+\gamma_{u} \Delta \hat{Z}_{u}^{\prime}-\lambda_{u} \Delta \hat{Z}_{u}^{\prime \prime}+\tilde{X}_{u}\right)-\phi\left(X_{u-}+\tilde{X}_{u}\right)\right) \\
& \left.-\left(\gamma_{u} \Delta \hat{Z}_{u}^{\prime}-\lambda_{u} \Delta \hat{Z}_{u}^{\prime \prime}\right) \phi^{\prime}\left(X_{u-}+\tilde{X}_{u}\right)\right)
\end{aligned}
$$

Taking then expectation, we obtain

$$
\begin{aligned}
\mathbb{E}\left[J_{s, t}^{X}\right]= & \mathbb{E}\left[\int _ { s } ^ { t } \int _ { - \infty } ^ { + \infty } \int _ { - \infty } ^ { + \infty } \left(\phi\left(X_{u}+\tilde{X}_{u}+\gamma_{u} x-\lambda_{u} y\right)-\phi\left(X_{u}+\tilde{X}_{u}\right)\right.\right. \\
& \left.\left.-\left(\gamma_{u} x-\lambda_{u} y\right) \phi^{\prime}\left(X_{u}+\tilde{X}_{u}\right)\right) \sigma_{\hat{Z}^{\prime}, \hat{Z}^{\prime \prime}}(d u, d x, d y)\right]
\end{aligned}
$$

where $\sigma_{\hat{Z}^{\prime}, \hat{Z}^{\prime \prime}}$ is the $\mathcal{F}$-dual predictable projection of the bivariate process $\left(\hat{Z}^{\prime}, \hat{Z}^{\prime \prime}\right)$. Since its coordinates are independent and have the same distribution as $Z^{\prime}, Z^{\prime \prime}$, the measure $\sigma_{\hat{Z}^{\prime}, \hat{Z}^{\prime \prime}}$ is the tensor product of the Lebesgue measure on $\mathbb{R}_{+}$and the two-dimensional Lévy measure concentrated on the axis and with two marginals $\nu_{+}$. Therefore we get from the above computations,

$$
\begin{aligned}
\mathbb{E}\left[J_{s, t}^{X}\right]= & \mathbb{E}\left[\int_{s}^{t} \int_{0}^{+\infty}\left(\phi\left(X_{u}+\tilde{X}_{u}+\gamma_{u} x\right)-\phi\left(X_{u}+\tilde{X}_{u}\right)-\gamma_{u} x \phi^{\prime}\left(X_{u}+\tilde{X}_{u}\right)\right) d u \nu_{+}(d x)\right] \\
& +\mathbb{E}\left[\int_{s}^{t} \int_{0}^{+\infty}\left(\phi\left(X_{u}+\tilde{X}_{u}-\lambda_{u} y\right)-\phi\left(X_{u}+\tilde{X}_{u}\right)+\lambda_{u} y \phi^{\prime}\left(X_{u}+\tilde{X}_{u}\right)\right) d u \nu_{+}(d y)\right] \\
= & \mathbb{E}\left[\int_{s}^{t} \int_{0}^{+\infty}\left(\phi\left(X_{u}+\tilde{X}_{u}+a\right)-\phi\left(X_{u}+\tilde{X}_{u}\right)-a \phi^{\prime}\left(X_{u}+\tilde{X}_{u}\right)\right) \gamma_{u}^{\alpha} d u \frac{d a}{|a|^{\alpha+1}}\right] \\
& +\mathbb{E}\left[\int_{s}^{t} \int_{-\infty}^{0}\left(\phi\left(X_{u}+\tilde{X}_{u}+b\right)-\phi\left(X_{u}+\tilde{X}_{u}\right)-b \phi^{\prime}\left(X_{u}+\tilde{X}_{u}\right)\right) \lambda_{u}^{\alpha} d u \frac{d b}{|b|^{\alpha+1}}\right],
\end{aligned}
$$

where in the last line we used two obvious changes of variables. Hence we obtain

$$
\begin{aligned}
\mathbb{E}\left[J_{s, t}^{X}\right]= & \mathbb{E}\left[\int_{s}^{t} \int_{-\infty}^{+\infty}\left(\phi\left(X_{u}+\tilde{X}_{u}+x\right)-\phi\left(X_{u}+\tilde{X}_{u}\right)-x \phi^{\prime}\left(X_{u}+\tilde{X}_{u}\right)\right)\right. \\
& \left.\times\left(\gamma_{u}^{\alpha} 1_{\{x>0\}}+\lambda_{u}^{\alpha} 1_{\{x<0\}}\right) d u \frac{d x}{|x|^{\alpha+1}}\right] .
\end{aligned}
$$

Certainly, a similar method applied in the backward case allows us to get

$$
\begin{aligned}
\mathbb{E}\left[J_{s, t}^{\tilde{X}}\right]= & \mathbb{E}\left[\int_{s}^{t} \int_{-\infty}^{+\infty}\left(\phi\left(\tilde{X}_{u}+X_{u}+x\right)-\phi\left(\tilde{X}_{u}+X_{u}\right)-x \phi^{\prime}\left(\tilde{X}_{u}+X_{u}\right)\right)\right. \\
& \left.\times\left(\tilde{\gamma}_{u}^{\alpha} 1_{\{x>0\}}+\tilde{\lambda}_{u}^{\alpha} 1_{\{x<0\}}\right) d u \frac{d x}{|x|^{\alpha+1}}\right] .
\end{aligned}
$$


Finally taking expectation in the Itô formula above and plugging the previous calculations, we have

$$
\begin{aligned}
\mathbb{E}\left[\phi\left(X_{t}+\tilde{X}_{t}\right)\right]-\mathbb{E}\left[\phi\left(X_{s}+\tilde{X}_{s}\right)\right] \\
=\mathbb{E}\left[\int_{s}^{t} \int_{0}^{+\infty}\left(\phi\left(X_{u}+\tilde{X}_{u}+x\right)-\phi\left(X_{u}+\tilde{X}_{u}\right)-x \phi^{\prime}\left(X_{u}+\tilde{X}_{u}\right)\right)\left(\gamma_{u}^{\alpha}-\tilde{\gamma}_{u}^{\alpha}\right) d u \frac{d x}{|x|^{\alpha+1}}\right] \\
\quad+\mathbb{E}\left[\int_{s}^{t} \int_{-\infty}^{0}\left(\phi\left(X_{u}+\tilde{X}_{u}+x\right)-\phi\left(X_{u}+\tilde{X}_{u}\right)-x \phi^{\prime}\left(X_{u}+\tilde{X}_{u}\right)\right)\left(\lambda_{u}^{\alpha}-\tilde{\lambda}_{u}^{\alpha}\right) d u \frac{d x}{|x|^{\alpha+1}}\right],
\end{aligned}
$$

which is non-positive by the convexity of $\phi$ and our assumptions. The proof of Theorem 2.2 is now complete.

To achieve this short note, it remains to prove our main result Theorem 2.1 on the basis of Theorem 2.2 .

Proof of Theorem 2.1. The proof is a straightforward consequence of Theorem 2.2 since under the conditions $\tilde{c}_{+}=c_{+}$and $\tilde{c}_{-}=c_{-}$and when the process $\tilde{K}$ is actually deterministic (equal to the given function $k$ ), the backward stable stochastic integral $\int_{0}^{T} \tilde{K}_{t} \tilde{d} \tilde{Z}_{t}$ has the same distribution as the forward integral $\int_{0}^{T} \tilde{K}_{t} d Z_{t}$.

Acknowledgments. The authors thank Yutao Ma for useful discussions. They are also grateful to the French Agency Campus France for financial support.

\section{REFERENCES}

[1] M. Arnaudon, J.C. Breton and N. Privault. Convex ordering for random vectors using predictable representation. Potential Anal., 29:327-349, 2008.

[2] J. Bergenthum and L. Rüschendorf. Comparison of option prices in semimartingale models. Finance Stoch., 10:222-249, 2006.

[3] J. Bergenthum and L. Rüschendorf. Comparison of semimartingales and Lévy processes. Ann. Probab., 35:228-254, 2007.

[4] M. V. Boutsikas and E. Vaggelatou. On the distance between convex-ordered random variables, with applications. Adv. in Appl. Probab., 34:349-374, 2002.

[5] J.C. Breton, B. Laquerrière and N. Privault. Convex comparison inequalities for nonMarkovian stochastics integrals. Stochastics, 85:789-806, 2013.

[6] J.C. Breton and N. Privault. Convex comparison inequalities for exponential jump-diffusion processes. Commun. Stoch. Anal., 1:263-277, 2007.

[7] J.C. Breton and N. Privault. Bounds on option prices in point process diffusion models. Int. J. Theor. Appl. Finance, 11:597-610, 2008.

[8] N. El Karoui, M. Jeanblanc-Picqué and S.E. Shreve. Robustness of the Black and Scholes formula. Math. Finance, 8:93-126, 1998.

[9] W. Hoeffding. On the distribution of the number of successes in independent trials. Anal. Math. Statist. 27:713-721, 1956.

[10] W. Hoeffding. Probability Inequalities for sums of bounded random variables. J. Amer. Statist. Assoc., 58:13-30, 1963.

[11] A. Joulin. On maximal inequalities for stable stochastic integrals. Potential Anal., 26:57-78, 2007. 
[12] O. Kallenberg. Some time change representations of stable integrals, via predictable transformations of local martingales. Stochastic Process. Appl., 40:199-223, 1992.

[13] T. Klein, Y. Ma and N. Privault. Convex concentration inequalities and forward-backward stochastic calculus. Electron. J. Probab., 11:486-512, 2006.

[14] B. Laquerrière and N. Privault. Deviation inequalities for exponential jump-diffusion processes. Theory Stoch. Process., 16:67-72, 2010.

[15] A. Müller and D. Stoyan. Comparison methods for stochastic models and risks. Wiley Series in Probability and Statistics. John Wiley and Sons, Chichester, 2002.

[16] J. Rosiński and W.A. Woyczyński. On Itô stochastic integration with respect to $p$-stable motion: inner clock, integrability of sample paths, double and multiple integrals. Ann. Prob., 14:271-286, 1986.

[17] L. Rüschendorf. On a comparison result for Markov processes. J. Appl. Probab., 45:279-286, 2008.

[18] L. Rüschendorf and V. Wolf. Comparison of Markov processes via infinitesimal generators. Statist. Decisions, 28:151-168, 2011.

[19] M. Shaked and J.G. Shanthikumar. Stochastic Orders. Springer Series in Statistics. Springer, New York, 2007.

(A. Joulin, corresponding author) UMR CNRS 5219, Institut de MathÉmatiques De Toulouse, Université de Toulouse, France

E-mail address: mailto:ajoulin(at)insa-toulouse.fr

(S.M. Manou-Abi) UMR CNRS 5219, Institut de Mathématiques de Toulouse, UniVersité De Toulouse, France

E-mail address: mailto:Manou.Mawaki(at)math.univ-toulouse.fr 